\title{
Barreras a la accesibilidad para la atención odontológica en parajes de la región del lberá
}

Accessibility barriers to dental care in places

of the region of Iberá
Barreiras de acessibilidade aos cuidados dentários em locais da região de lberá
Fecha de Recepción

17 de noviembre de 2014
Aceptado para su publicación

I5 de diciembre de 2014
Prof. María Cristina Ojeda Subdirectora. Prof. Titular Cátedra de Odontología Social y Comunitaria

Prof. Roque Oscar Rosende Director. Prof. Titular Cátedra de Cirugìa Maxilo Facial

Od. María florencia Maldonado Seniquel Auxiliar Docente. Cátedra de Microbiología

Lugar de Trabajo Facultad de Odontología. Avda Libertad 5450 Tel./fax: 3783-57992

Código postal: 3400

\section{Resumen}

El presente trabajo describe los resultados parciales de la relación entre usuarios y servicios, en cuanto a la cobertura, accesibilidad y disponibilidad a la atención de salud bucal, de las familias habitantes de seis parajes de la región de los Esteros del lberá.

Las variables analizadas son dimensiones en la investigación del componente bucal de la salud del hombre del lberá.

El objetivo es describir la relación funcional entre la población, profesionales, equipamiento e insumos, manifestada ya sea en obstáculos, impedimentos y dificultades, o bien, factores facilitadores para las familias que demanden atención odontológica, de tal manera de posibilitar a las poblaciones de zonas rurales, un acceso equitativo a los servicios de salud.

\section{Palabras claves}

Disponibilidad, Necesidad de Salud, Aceptabilidad, Distancias, Continuidad.

\section{Summary}

This paper describes partial results on the relationship between users and services, considering coverage, accessibility and availability of oral health care, in families inhabitants of the lbera region.

The variables are dimensions of analysis in the investigation of oral health component in Ibera people. 
The principal aim is describe the functional relationship between population, professionals, equipment, supplies and facilitating factors for families who require dental care, in order that rural populations have access to an equitable health services.

\section{Key words}

Vailability, Health need, Acceptability, Distances, Continuity.

\section{Resumo}

Este artigo descreve os resultados parciais da relação entre usuários e serviços, considerando cobertura, acessibilidade e disponibilidade aos cuidados de saúde oral, em famílias habitantes na região dos pântanos lbera.

As variáveis são dimensões de análise de componente na investigação de saúde bucal em homem de lbera.

O objetivo é descrever a relação funcional entre população, profissionais, equipamentos e acessórios, se manifesta obstáculos, impedimentos e dificuldades, ou, facilitando fatores para famílias exigentes atendimento odontológico, por assim permitir as populações rurais, 0 acesso equitativo aos serviços de saúde.

\section{Palavras chave}

Disponibilidade, Necessidade de saúde, Aceitabilidade, Distâncias, Continuidade.

\section{Introducción}

La orientación de nuevas estrategias en salud, ubican a los sistemas de prestación de servicios en la búsqueda de modelos que mejoren la calidad de vida y que brinden mejor eficiencia y efectividad. Entre algunos de los elementos innovadores a introducirse dentro de este concepto, está la urgente necesidad de proporcionar a la población rural, hasta la actualidad postergada, programas odontológicos considerando los contextos socio-culturales y las barreras ecológicas'.

Lo anterior se debe a que este importante subgrupo poblacional no ha sido considerado como problema de salud pública, puesto que sólo se ha tomado en cuenta la morbilidad y mortali- dad dentaria, sin estimar los riesgos prevenibles, los que deberían ser evaluados con indicadores consensuados ${ }^{2}$.

La descripción de éste estudio, busca identificar la existencia de barreras potenciales de acceso a la salud bucal en la región del iberá, considerando no solo las geográficas, sino otras diferentes situaciones como ser la cultura, el lenguaje, los ingresos, la ubicación y complejidad de los centros de atención.

\section{Material y métodos}

Es un estudio epidemiológico, observacional descriptivo de corte transversal.

Se realizó una muestra por conveniencia en 6 parajes de la región del lberá: Uguay, Dto. Mercedes; Cambá Trapo, Dto. Gral San Martín; Pje libertad, Dto. ituzaingó; Los Lapachos, Dto. Concepción; Galarza, Dto. Sto. Tomé; Montaña, Dto. San Miguel.

La información la suministraron informantes claves: maestros, directores y supervisoras de escuelas, agentes de policía, administrativos, enfermeros, agentes sanitarios, guardaparques, médicos, odontológos y familias de los parajes.

Se realizaron entrevistas con una guía de preguntas abiertas y sondeos neutros no dirigidos, a fin de obtener información sobre diferentes aspectos de la salud.

Los centros para la obtención de los datos fueron visita en los domicilios y escuelas.

Las respuestas se registraron en cuaderno de campo y se agruparon en matrices, según datos emergentes y recurrentes sobre las siguientes categorías: distancia geográfica a los centros de salud, estado de las rutas y caminos, medios de transporte, números de profesionales, días y horarios de atención, equipamiento, disponibilidad de insumos e instrumental y frecuencia de visitas a los parajes, necesidad de atención y odontológica y solicitud de atención.

\section{Resultados parciales}

La región de los Esteros del lberá, se caracteriza por la presencia de amplias zonas rurales de población dispersa, demograficamente son de baja densidad - menos de $30 \mathrm{hab} / \mathrm{Km}^{2}$-, o bien residentes en asentamientos pequeños de 5 a 


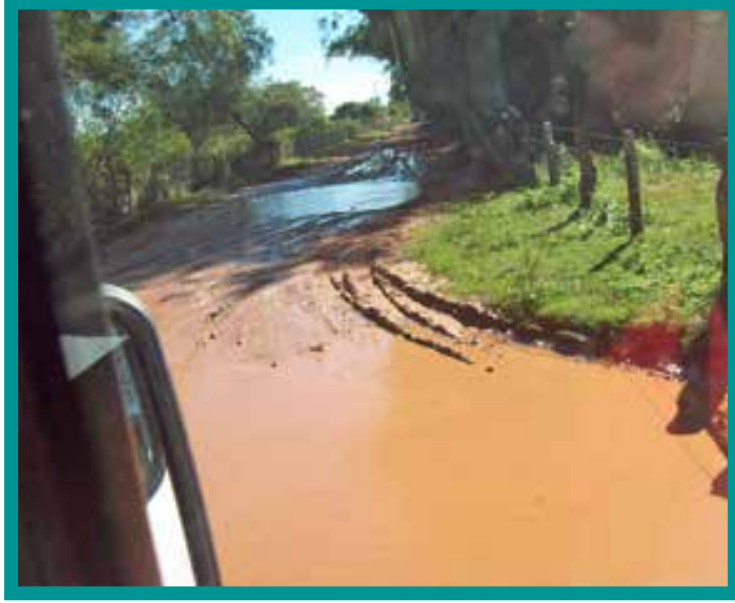

Estado de las rutas y caminos Pje Montaña.

7 familias, que suman entre 20 a 25 habitantes.

Se realizaron 150 encuestas a informantes claves y familias.

De la información vertida en la matriz de datos se destaca:

90\% de familias desvinculadas entre sí, con situación situación de incomunicación.

$85 \%$ de rutas y caminos intransitables y transportes inadecuados.

$100 \%$ de ausencia de odontológos.

No se registraron salas ni centros de salud, siendo los más cercanos entre 30 a $40 \mathrm{Km}$. de distancia.

En cuanto a los pobladores, la concepción de salud está relacionada a lo curativo, poseen una marcada resignación a la enfermedad, asisten a la consulta ante el dolor, con el cual conviven, y consideran que las enfermedades bucales no se pueden evitar, y se deben a "dientes débiles", con la exodoncia como única solución, siendo además la única práctica que se ofrece en los servicios de atención a los que tienen acceso.

El $90 \%$ de los encuestados, manifiestaron miedo al odontólogo por el instrumental que utiliza.

\section{Discusión de los resultados}

El aislamiento geográfico y el mal estado de las rutas, es considerada la mayor de las dificultades, siguiendo en importancia, la ausencia de odontológos y médicos, incumplimiento de los días y horarios de atención en los servicios de los pueblos.

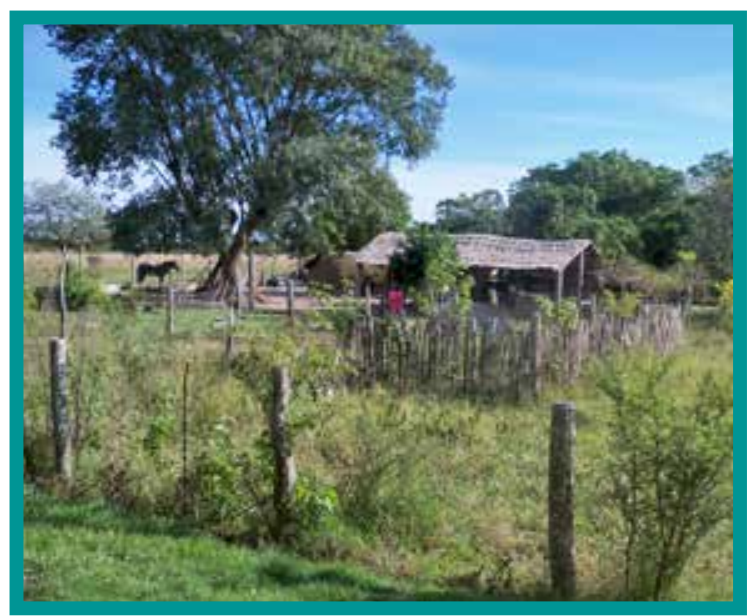

Vivienda uguay.

En consecuencia, éstas poblaciones son consideradas de alto riesgo para las enfermedades bucales, debido al poco acceso que tienen a los servicios de salud y a prácticas de higiene bucal inadecuadas, probablemente influidas por el contexto sociocultural en el cual están inmersos. ${ }^{3}$

Además, los trámites burocráticos, la cita previa, las recetas, las derivaciones y las distancias a los centros de mayor complejidad, suscita una oferta de servicios que no facilita el acceso al derecho equitativo a la salud ${ }^{4}$.

Por esta razón, es necesario implementar un sistema de vigilancia y acompañamiento que permita promover la salud en su componente bucal $^{5}$, en procura de responder en tiempo y lugar adecuado, con talento humano en número y disponibilidad suficiente para toda la población como garantía de calidad y acceso a un goce pleno del derecho a la salud con equidad ${ }^{6}$, porque la salud es un derecho de la población y es responsabilidad del estado ${ }^{7}$.

\section{Conclusión}

Entre las barreras para la accesibilidad a la atención de salud bucal en la región del lberá, cabe destacar que el odontólogo es una figura ajena a la realidad cotidiana, aunque sienten la necesidad de su práctica.

El miedo, sumado a su ausencia de profesionales, de salas de atención, así como la carencia de recursos para asistir a centros de mayor complejidad, las distancias geográficas y el descono- 


\section{8}

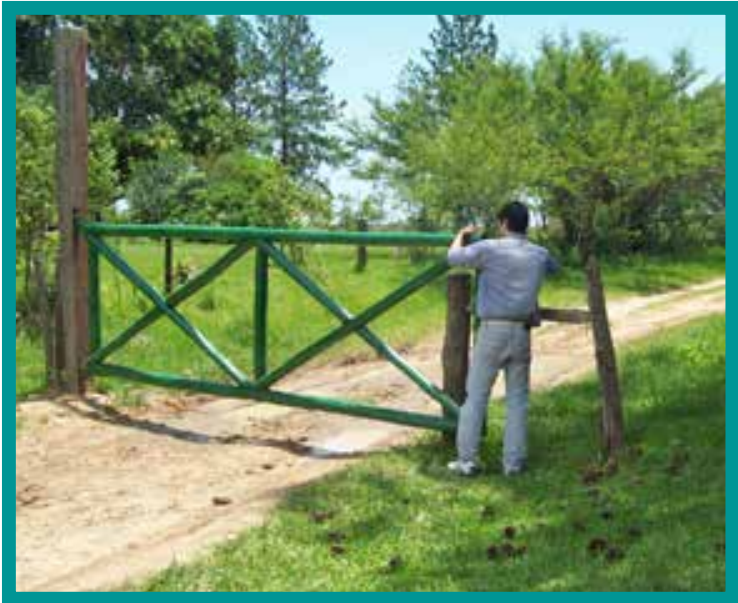

Solicitud de permisos para el ingreso a los parajes. Terrenos privatizados.

cimiento de sus derechos, motiva la falta total de controles odontológicos, con la consecuente alta prevalencia de patologías bucodentales.

Para enfocar la solución de los problemas de salud, es necesario identificar las necesidades, las demandas y las espectativas sociales, como así también los cambios necesarios en la organización del sector salud y en el financiamiento, para promover prácticas con sólida base científica, orientación preventiva y participación social.

\section{Bibliografía}

I. OPS. Indicadores de Salud: Elementos básicos para el análisis de la situación de salud. Boletín Epidemiológico, 22 (4): Diciembre 200 I.

2. Piovano S; Squassi A ; Bordoni N. Estado del Arte de Indicadores para la Medición de Caries Cental http://www.odon.uba.ar/ revista/2010vol25num58/docs/piovano.pdf Acceso 25/09/20l4.

3. Fernández González C, Núñez Franz L, Díaz Sanzana N. Determinantes de Salud oral en Población de 12 años. Rev. Clin. Periodoncia Implantol. Rehabil. Oral Vol. 4(3); II7-I2I, 2011.

4. Ramos Ruiz, J. A., Pérez Milena, A., Enguix Martínez, N., Álvarez Nieto, C., \& Martínez Fernández, M. L. (20/3). Diagnóstico comunitario

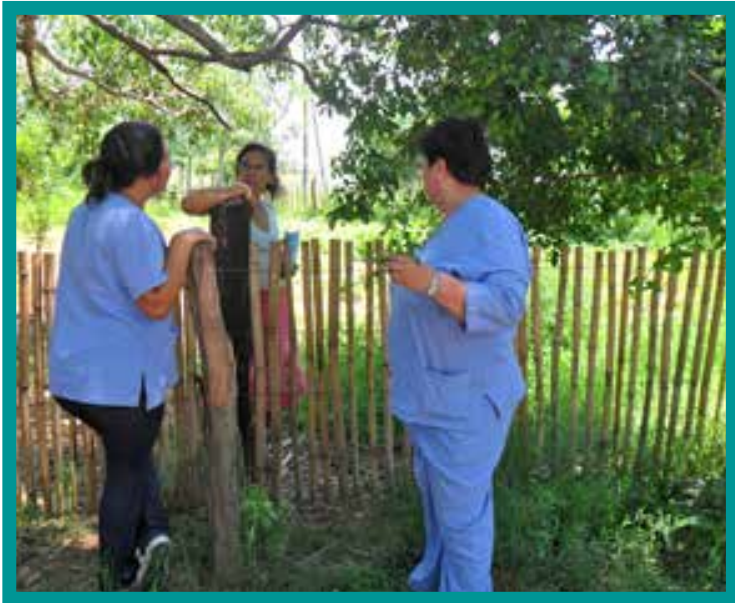

Entrevista a practicantes de medicina folclórica.

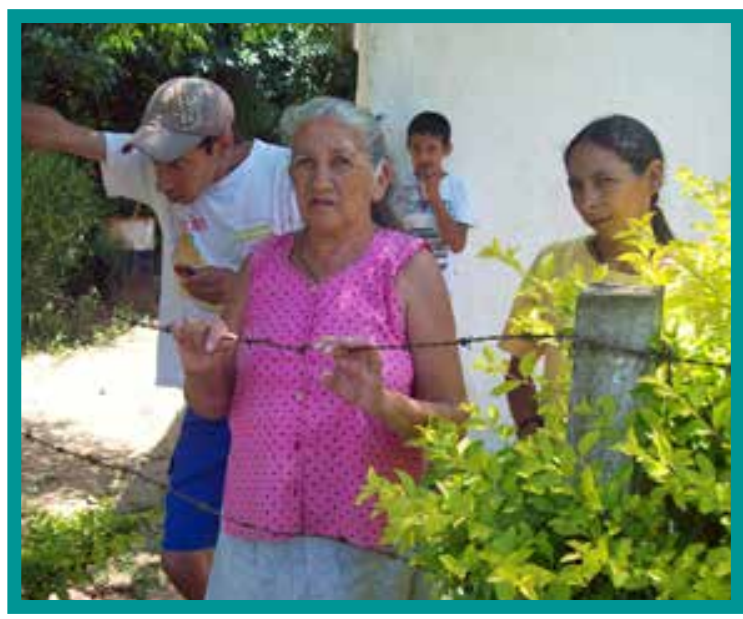

Visita a las familias.

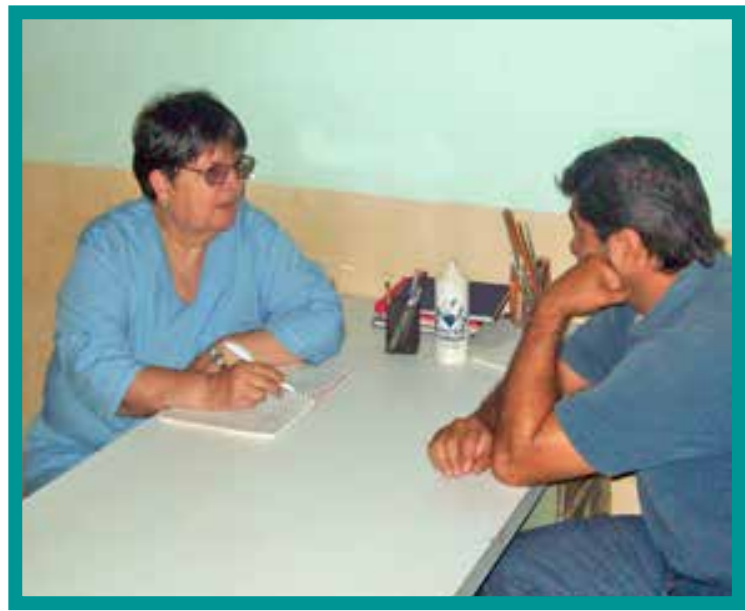

Entrevista a maestros rurales. 


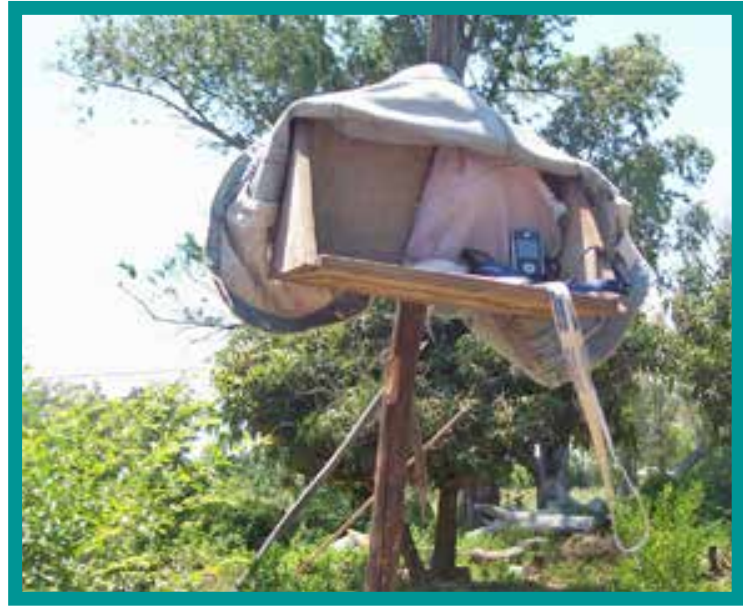

Celulares sin señal, ubicados en los àrboles.

mediante técnicas cualitativas de las expectativas $y$ vivencias en salud de una zona necesitada de transformación social. Atención Primaria, 45(7), 358-367.

5. Concha Sánchez SC, Morales Borrero C. La inequidad en el acceso a la atención odontológica de las mujeres gestantes en Santander. rev.univ. ind.santander.salud 21 04; 46 (I): 35-45

6. Organización Mundial de la salud. Informe sobre la salud en el mundo, la financiación de los sistemas de salud: el camino hacia la cobertura universal. Suiza: OMS; 2010.

7. Rivera Martínez César A. Salud Bucal en Niños Pre-escolares de una Zona Rural Chilena. Int. J. Odontostomat. [online]. 5(I):83-86, $201 \mathrm{I}$. Disponible en: http://www.scielo.cl/scielo. php?pid=S07/8. Acceso 29/1 I/0 /4.

8. Bradley E, Thompson JC, Access and quality of rural headthcare. Ethiopian millennium rural initiative. International Journal for quality in health care. 20I I Marzo 23(3)

9. Delgado B., i., Cornejo-ovalle, M., Jadue H., L., Huberman, J. Determinantes sociales y equidad de acceso en la salud dental en Chile. Cient. dent. 20I3; 10; 2: I0I-109.

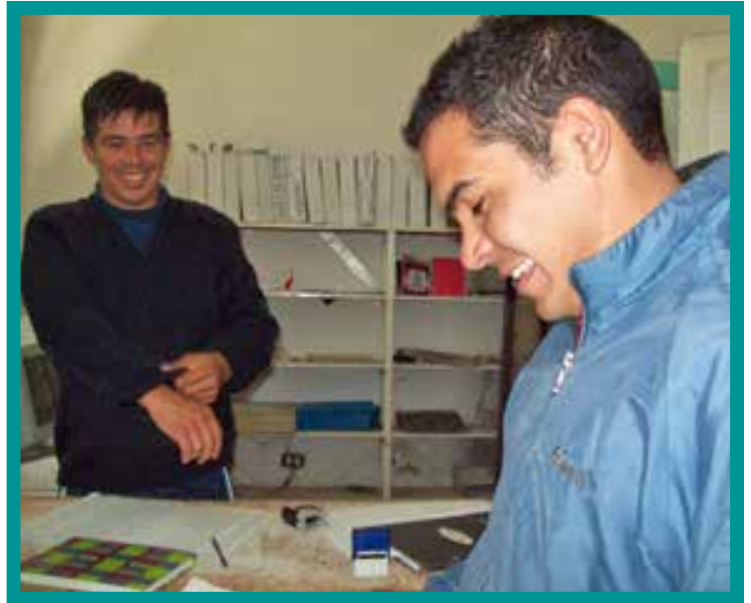

Entrevistas a agentes de policía.

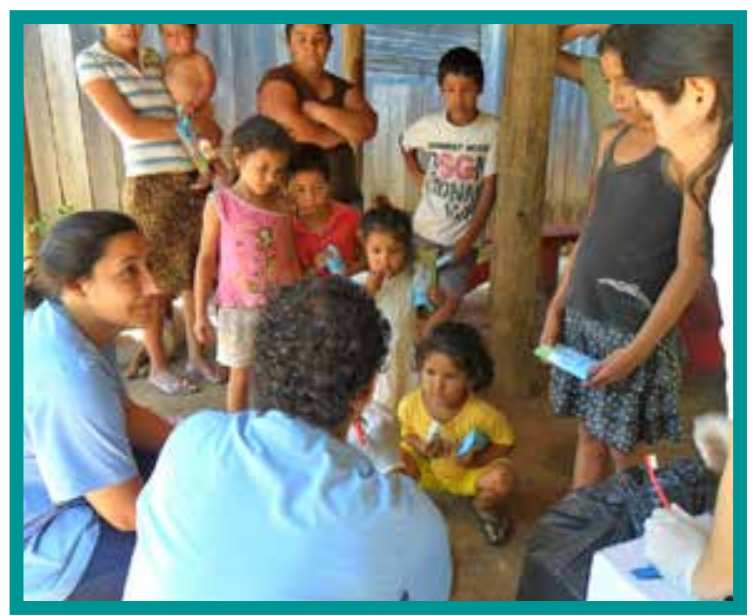

Motivaciòn a niños.

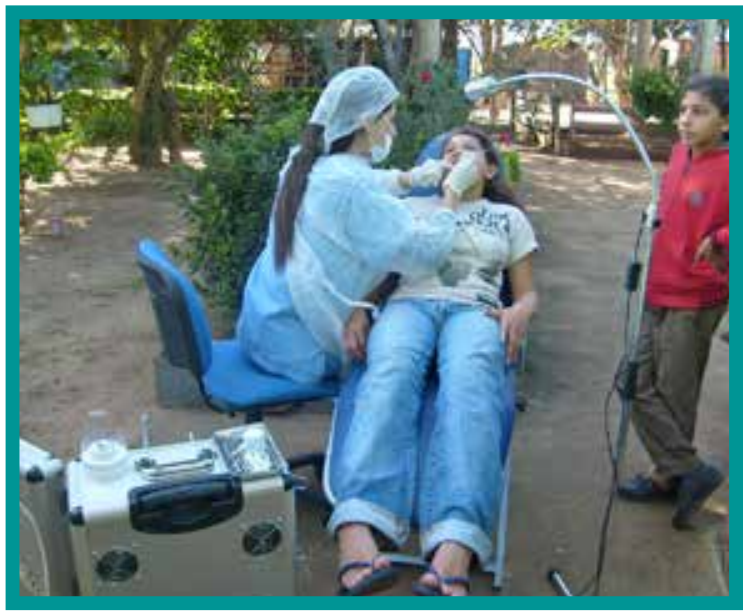

Atención clínica con equipo electrógeno familiar. Pje Libertad. 